\title{
Observations of magnetic and kinetic helicity proxies
}

\section{Hongqi Zhang}

Key Laboratory of Solar Activity, National Astronomical Observatories, Chinese Academy of Sciences, Beijing 100012

email: hzhang@bao.ac.cn

\begin{abstract}
The helicity is important to present the basic topological configuration of magnetic field in solar atmosphere. The distribution of magnetic helicity in solar atmosphere is presented by means of the observational (vector) magnetograms. As the kinetic helicity in the solar subatmosphere can be inferred from the velocity field based on the technique of the helioseismology and used to compare with the magnetic helicity in the solar atmosphere, the observational helicities provide the important chance for the confirmation on the generation of magnetic fields in the subatmosphere and solar dynamo models also. In this paper, we present the observational magnetic and kinetic helicity in solar active regions and corresponding questions, except the relationship with solar eruptive phenomena.
\end{abstract}

Keywords. Sun: activity - Sun: magnetic fields - Sun: helicity

\section{Importance and definition of magnetic helicity}

Helicities are topologically a measure of the structural complexity of the corresponding fields (Woltjer, 1958a,b). As indicated by Taylor (1986) that the topological invariants of ideal plasma so that only total magnetic helicity survives. Helicity is described in terms of the internal structure of a flux tube and the external relations between flux tubes.

The magnetic, current and kinetic helicity are defined as

$$
\begin{aligned}
H_{m} & =\int_{V} h_{m} d^{3} x=\int_{V} \mathbf{A} \cdot \nabla \times \mathbf{A} d^{3} x, \\
H_{c} & =\int_{V} h_{c} d^{3} x=\int_{V} \mathbf{B} \cdot \nabla \times \mathbf{B} d^{3} x \\
H_{k} & =\int_{V} h_{v} d^{3} x=\int_{V} \mathbf{u} \cdot \nabla \times \mathbf{u} d^{3} x
\end{aligned}
$$

where the vector potential A can not be observed immediately, while the magnetic field $\mathbf{B}$ and velocity field $\mathbf{u}$ is observational quantities normally. The mean helicity densities $\bar{h}_{(m, c, v)}$ have been normally used in some times. The helicity can be separated into two kinds. One is the self helicity, which relates to the magnetic or velocity flux tubes twisted themselves. This helicity may be used to analyze the twisted magnetic or velocity flux. Another is the mutual helicity, which relates to the different magnetic (or velocity) flux tubes linked each other. As the helicity contains both, the total helicity can be written in the form

$$
H=T \Phi^{2}+2 L \Phi_{1} \Phi_{2}
$$

where the $T$ is the twisted number of magnetic flux $\Phi$ and the $L$ is the linkage number of different flux $\Phi_{1}$ and $\Phi_{2}$. 
The relative change of magnetic helicity in the solar atmosphere can be inferred by the magnetic field across the boundary surface (Berger and Field, 1984)

$$
\frac{d H_{m}}{d t}=-2 \oint_{S}\left[\left(\mathbf{V}_{t} \cdot \mathbf{A}_{p}\right) B_{n}-\left(\mathbf{A}_{p} \cdot \mathbf{B}_{t}\right) V_{n}\right] d s,
$$

where the magnetic field $\mathbf{B}$ and velocity field $\mathbf{V}$ are observable in the solar atmosphere. The subscripts have their normal meanings. The first term in eq. (1.4) provides the contribution from the twisted motion of footpoints of magnetic field in the solar surface, while the second term does that from the emergence of twisted magnetic flux from the subatmosphere. This also has been analyzed by Démoulin and Berger (2003), Pariat, Démoulin and Berger (2005).

The current helicity density $h_{c}\left(h_{c}=\mathbf{B} \cdot \nabla \times \mathbf{B}\right)$ is another important physical quantity for the measure of the magnetic field in the solar atmosphere. It is noticed that only as $\nabla \times \mathbf{A}$ is parallel to $\mathbf{A}$ the relationship of between the magnetic and current helicity densities becomes simple, and both helicity density show the same sign constantly (Zhang, 2001). The relationship between the mean magnetic and current helicities is still probably a basic question in the statistical analysis of magnetic fields (Zhang, et al., 2012).

Moreover, it is found (Abramenko et al.,1996; Bao and Zhang, 1998) that only a part (vertical component) of current helicity density in the photosphere $h_{c z}=\mathbf{B} \cdot(\nabla \times \mathbf{B})_{z}$ can be inferred from the photospheric vector magnetograms, due to the observational limitation. A similar limitation can be found also in the analysis of the force free factor $\alpha=\frac{\mu J_{z}}{B_{z}}$ (Pevtsov et al., 1994), which also does not contain any information on the horizontal part of current helicity density. The mean helicity densities $\bar{h}_{c}$ (or mean force free factor $\bar{\alpha}$ ) are normally used to infer the handedness of fields quantitatively in active regions.

Subsurface kinetic helicity can be computed from subsurface flow velocities, which are readily available through the time-distance analysis pipeline in the heliosencemology technique (cf. Zhao et al. 2012).

It is suggested a dynamo mechanism in the solar interior based on the combined action of differential rotation and cyclonic convective vortices (Paker 1955) as a viable way to generate magnetic fields capable of driving the activity cycle. According to mean field dynamo theory, the electromotive force $\mathbf{E}$ averaged over convective eddies has a component parallel to the magnetic field, $\mathbf{E}=\alpha<\mathbf{B}>+\ldots$, where the pseudoscalar $\alpha$ is related to kinetic and electric current helicities $\left(\alpha^{k}\right.$ and $\left.\alpha^{c}\right)$ (cf. Brandenburg and Subramanian, 2005; Rädler and Rheinhardt, 2007; Sokoloff 2007). It can be written in the form

$$
\alpha=\alpha^{k}+\alpha^{c}=h_{k} \phi_{v}+\frac{\phi_{m}}{\rho} h_{c}
$$

where, $h_{k}$ and $h_{c}$ are proportional to the kinetic and current (or magnetic) helicities respectively and $\phi_{v}$ and $\phi_{c}$ are quenching functions.

The alpha effect is reevaluated in terms of ensemble-averaged properties of the magnetic fluctuation spectrum. It is proposed that the turbulent current helicity must be opposite in sign to the mean-field current helicity in order for the alpha effect to play a role in overcoming the resistive diffusion of large-scale magnetic fields. Pouquet, Frisch, and Leorat (1976) indicated that study of helicity is important in its kinetic and in its magnetic form for generation of large-scale magnetic fields by turbulence. Keinigs (1983) and Keinigs and Gerwin (1986) presented that in a magnetized plasma the alpha effect 
represents a turbulently generated electromotive force directed along the mean magnetic field. According to Kleeorin and Rogachevskii (1999) and Brandenburg and Subramanian (2005), the change of magnetic (current) helicity can been inferred in the form

$$
\frac{\partial h_{c}}{\partial t} \approx-\frac{2}{l^{2}}\left[<\mathbf{B}>\cdot \nabla \times<\mathbf{B}>-\left(\alpha_{k}+h_{c}\right)<\mathbf{B}>^{2}\right]
$$

where the symbols have their normal meanings. It means that the observational solar vector magnetograms can be statistically used to get the possible message on the generation of magnetic field inside of the Sun due to the solar dynamo. The determination of the kinetic helicity in the solar atmosphere is difficult, while the twist of magnetic fields can be estimated from photospheric vector magnetograms of solar active regions (Abramenko et al. 1996; Bao and Zhang 1998).

\section{Basic configuration and transfer of magnetic helicity in solar atmosphere}

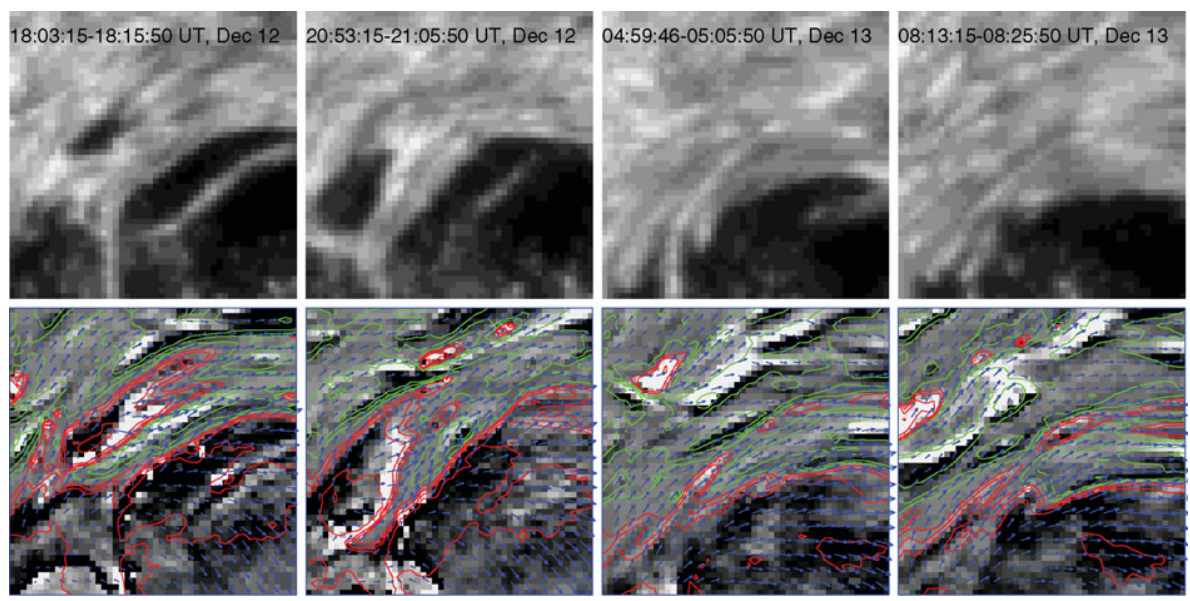

Figure 1. The monochromatic images (top) and vertical component of electric current helicity density (bottom) inferred by the local vector magnetic field in active region NOAA10930. The white (black) indicates the positive (negative) sign of the current helicity. Arrows show the transverse component, and the red and green contours show the longitudinal components of the magnetic field of $\pm 200,500,1000,2000,4000$ Gauss. The size of images is $8^{\prime \prime} \times 8^{\prime \prime}$. (Zhang, 2010)

Helicity in solar active regions has been noticed recently (cf. Seehafer, 1990; Pevtsov et al., 1994; Wang, 1996; López Fuentes et al., 2000; Deng et al., 2001; Yang et al., 2012; Xu et al., 2012). Chae (2001) showed how to observationally determine the rate of magnetic helicity transport via photospheric footpoint shuffling from a time series of line-of-sight magnetograms. From a series of photospheric-vector magnetograms and corresponding soft X-ray images, it is found (Zhang, 2001 and Zhang 2006a) that the newly emerging magnetic flux associates the current helicity from the subatmosphere in the active regions with the redistribution of the current helicity density in the upper atmosphere, i.e. it provides observational evidence that flux and helicity emerge together. Kusano et al. (2002) indicated that the photospheric shear motion and the flux emergence process have equally contributed to the helicity injection and have supplied magnetic helicity of opposite signs into the active regions. Liu and Zhang (2006) found that the 
rotation of photospheric footpoints forms in the earlier stage of magnetic flux emergence and the relative shear motion of different magnetic flux systems appears later in an active region. The similar case on the rotation of sunspots with the injection of helicity has been provided by Zhang, Liu and Zhang (2008). Jeorg and Chae (2007) indicated that the evolution of injective quantity of magnetic helicity depends on the developing phase of active regions.

In the analysis of fine helical features in the active regions, it is found the patches of positive and negative helicities were intermixed showing a mesh pattern in the sunspot umbra and a thread pattern in the penumbra ( $\mathrm{Su}$ et al., 2009). The fine distributions of $\alpha_{z}$ and $h_{c}$ on a penumbral filament indicated that it may be possible for the two opposite helicities to coexist in a filament and their magnitudes were nearly equivalent. It is found (Zhang, 2010) that the individual magnetic fibrils are dominated by the current density component caused by the magnetic inhomogeneity, while the large-scale magnetic region is generally dominated by the electric current component associated with the magnetic twist in Figure 1. The current mainly flows around the magnetic flux fibrils in the active regions. Venkatakrishnan and Tiwari (2009) pointed out that the existence of a global twist for a sunspot even in the absence of a net current is consistent with a fibril-bundle structure of the sunspot magnetic fields. Moreover, Tian and Alexander (2009) indicated from this statistical study that the leading (compact) polarity injects several times more helicity flux than the following (fragmented) one (typically 3-10 times).

\section{Relationship between magnetic helicity and solar cycles}

\subsection{Hemispheric rule of magnetic (current) helicity}

Hale et al. (1919) firstly discovered that $\mathrm{H} \alpha$ penumbral features show the direction of whirl in the Northern hemisphere is left-handed or anti-clockwise, while in the Southern hemisphere it is right-handed or clockwise. Ding, Hong and Wang (1987) statistically analyzed the distribution of spiral patterns in the southern and northern hemispheres and believed that the differential rotation may be a fundamental solar dynamo for the formation of the spiral spots. Based on above hemispheric rule Seehafer (1990), Pevtsov, Canfield and Metcalf (1995) found in their data set, $76 \%$ of the active regions in the northern hemisphere have negative helicity, and $69 \%$ in the southern hemisphere, positive. It is roughly consistent with the statistical results on the handedness of spiral sunspots by Ding, Hong and Wang (1987). The soft X-ray loops in the solar atmosphere also provide the signatures of the handedness of magnetic fields (Rust and Kumar, 1996). Moreover, Abramenko, Wang and Yurchishin (1996), Bao and Zhang (1998) and Hagino and Sakurai (2004) studied the current helicity of solar active regions inferred from the vector magnetograms of active regions and found the similar hemispheric trends of helicity also. The confirmation on the hemispheric sign rule of large-scale helicity has been done by Pevtsov and Latushko (2000) and Wang and Zhang (2010) from full disk magnetograms observed by MDI/SOHO. Moreover, Zhang (2006b) reported that the statistical analysis of strong fields gives a result: both $\alpha$ and current helicity present a sign opposite to that of weak fields in the active regions. The distinguishability between the weak and strong field relates to the basic question on the analysis of vector magnetograms in the active regions.

As following the injection of magnetic helicity from active regions, LaBonte, Georgoulis and Rust (2007) proposed that the weak hemispherical preference of helicity injection, positive in the south and negative in the north, is caused by the solar differential rotation. Tian et al. (2001) found that there is a negative correlation between the sign of the tilt 

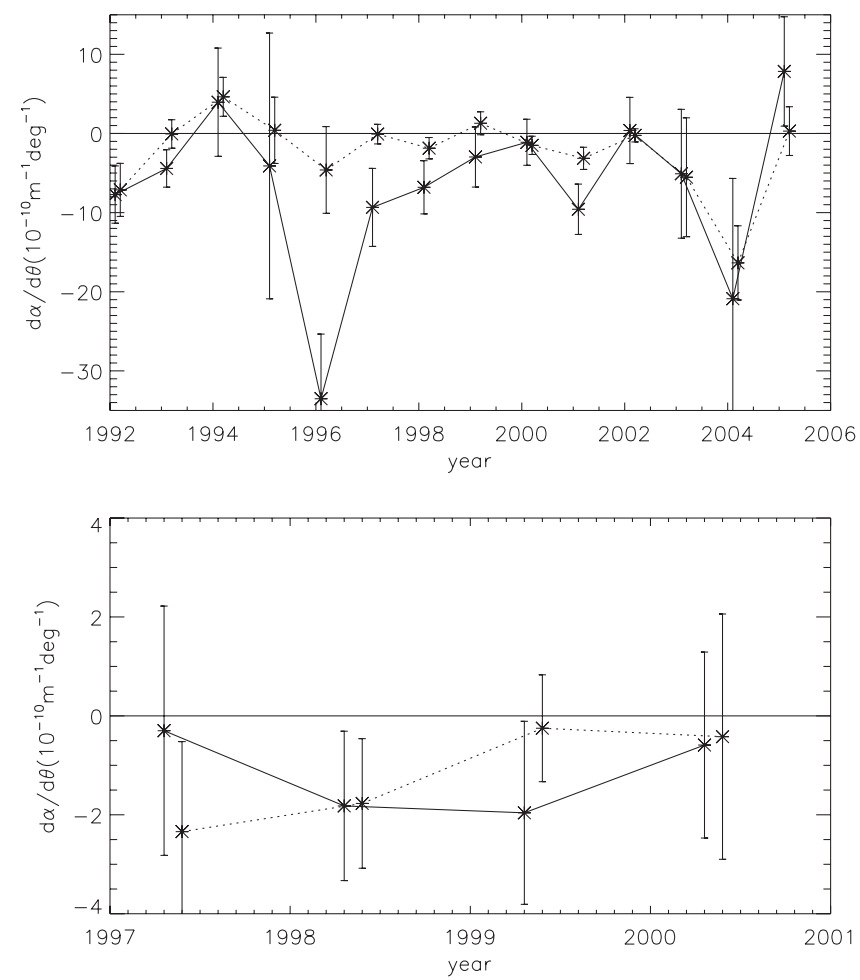

Figure 2. Comparison on the statistical variation of mean $\alpha$ (helicity parameter) of same solar active regions with latitude from different data sets between Huairou Solar Observing Station of National Astronomical Observatories of China (solid line) and National Astronomical observatory of Japan (dash line) (top), and also that between Mees Solar Observatory (solid line) and National Astronomical observatory of Japan (dash line) (bottom). (It is the same to the results by $\mathrm{Xu}$ et al., 2007)

angle and the sign of the current helicity Yang, Zhang and Büchner, (2009) investigated the accumulation of helicity in newly emerging simple bipolar solar active regions. It is found that the accumulated helicity is proportional to the exponent of magnetic flux $\left(|H| \propto \Phi^{1.85}\right)$ in the 58 selected newly emerged simple ARs. $74 \%$ of ARs have a negative (positive) helicity when the above defined tilt angle rotates clockwise (counter-clockwise). This means that the accumulated helicity and writhe have the same sign for most of the investigated ARs according to the tilt angle evolution of ARs.

For estimating the accuracy on the measurements of current helicity in the solar active regions, Pevtsov, Dun and Zhang (2006) used 270 pairs of vector magnetograms observed at different observatories. A comparison also with a series of helicity parameters (force free $\alpha$ ) from photospheric vector magnetograms observed by the Solar Flare Telescope (SFT) at Mitaka (MTK) of the National Astronomical Observatory of Japan has been taken by $\mathrm{Xu}$ et al.(2007) in Figure 2. It is consistent with the results of Hagino and Sakurai (2005) on the analysis of hemispheric magnetic helicity rule with solar cycles. Moreover, Pevtsov et al. (2008) concluded that because the hemispheric helicity rule is a weak tendency with significant scatter, an annual subset of active regions is likely to produce statistically unreliable results. 


\subsection{Evolution of magnetic (current) helicity with solar cycle}

By comparing the relationship between the helicity and solar dynamo models by by Seehafer (1994), Rüdiger, Pipin and Belvedére (2001) and Brandenburg, Dobler and Subramanian (2002), Longcope, Fisher and Pevtsov (1998) discussed the flux-tube twist resulting from helical turbulence. This process, designated the Sigma-effect, operates on isolated magnetic flux tubes subjected to buffeting by turbulence with a nonvanishing kinetic helicity.

Based on the observational current helicity of solar active regions with the possible formation depth in the solar convective zone (SCZ) (Kuzanyan et al., 2003), Kleeorin et al. (2003), Zhang et al. (2006), Sokoloff et al., (2008) and Zhang et al. (2012) attempted to connect observational data on current helicity in solar active regions with solar dynamo models. The predictions of this model about the radial distribution of solar current helicity tend to be in remarkable agreement with the available observational data; in particular the relative volume occupied by the current helicity of 'wrong' sign grows significantly with the depth.

Zhang and Bao (1998) analyzed the latitudinal distribution of the photospheric current helicity for active regions, including most of the large ones observed in the period of 1988-1997. It is found that the negative maximum values of current helicity occurred in 1989 and 1991, while those positive around 1992. Bao, Ai and Zhang (2000) computed the sign of different current helicity parameters (i.e. $\alpha_{b e s t}$ and $h_{c}$ ) for active regions during the rise of solar cycle 23 . The results indicate the $59 \%$ the active regions in the northern hemisphere have negative $\alpha_{b e s t}$ and $65 \%$ in the southern hemisphere have positive. However, the helicity parameter $h_{c}$ shows a weaker opposite hemisphereic preference in the new solar cycle. Hagino and Sakurai (2005) found that although the hemispheric sign rule of helicity generally holds, it is found significant time variations in the yearly values of helicity during the observation period. The hemispheric sign rule of helicity is satisfied in the solar maximum phase, but may not be so in the solar minimum phase.

Choudhuri, Chatterjee and Nandy (2004) calculated helicities of solar active regions based on the idea that poloidal flux lines get wrapped around a toroidal flux tube rising through the convection zone, thereby giving rise to the helicity. They found that during a short interval at the beginning of a cycle, helicities tend to be opposite of the preferred hemispheric trends. Xu et al. (2009) studied the behavior of the electric-current and magnetic helicities in the course of the solar-activity cycle in the framework of Parker's very simple model for the solar dynamo. They proposed a possibility of the reverse of hemispheric helicity rule in the end of solar cycle. These are basically consistent with observational tendency by Bao, Ai and Zhang (2000), Hagino and Sakurai (2005) and $\mathrm{Xu}$ et al. (2007).

The statistical imbalance of magnetic helicity of solar active regions in both hemispheres with solar cycles has discovered by Zhang et al. (2010a) recently, who analyzed a series of vector magnetograms of solar active regions observed at Huairou Solar Observing Station in China for more than 20 years. They found the following observational evidence: magnetic (electric current) helicity and twist patterns are, in general, antisymmetric with respect to the solar equator. The helicity pattern is more complicated than Hale polarity law for sunspots. Areas of the "wrong" sign have been found at the ends of the butterfly wings as well as at their very beginnings. The maximum value of helicity, at the surface at least, seems to occur near the edges of the butterfly diagram of sunspots. It is consistent basically with the results by Tiwari et al.(2009) based on the analysis of 43 sunspots in a period of solar cycle. The handedness of large scale soft X-ray loops nearby solar active regions with solar cycle was analyzed by Zhang et al. 


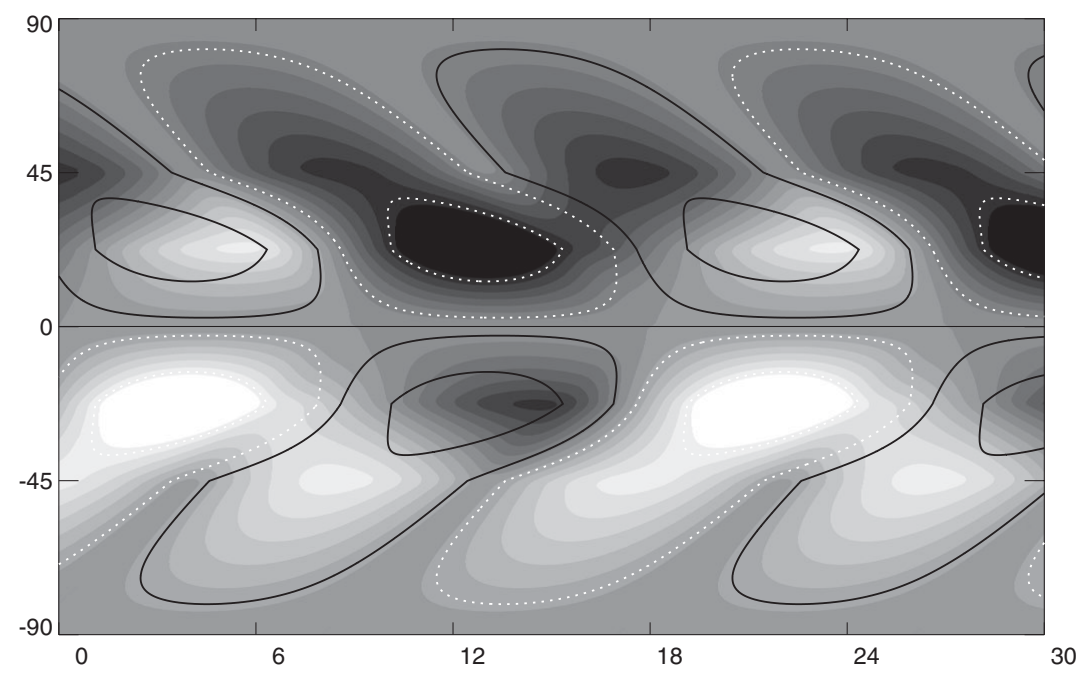

Figure 3. Latitude - time distribution of the magnetic helicity (gray scale) superposed onto the analogous distribution of the toroidal magnetic field (contours; the solid and dashed contours correspond to positive and negative values of $B$ ). The dimensionless time is plotted on the horizontal axis. The phase difference of between the magnetic field and helicity has been ignored. (Zhang, 2012)
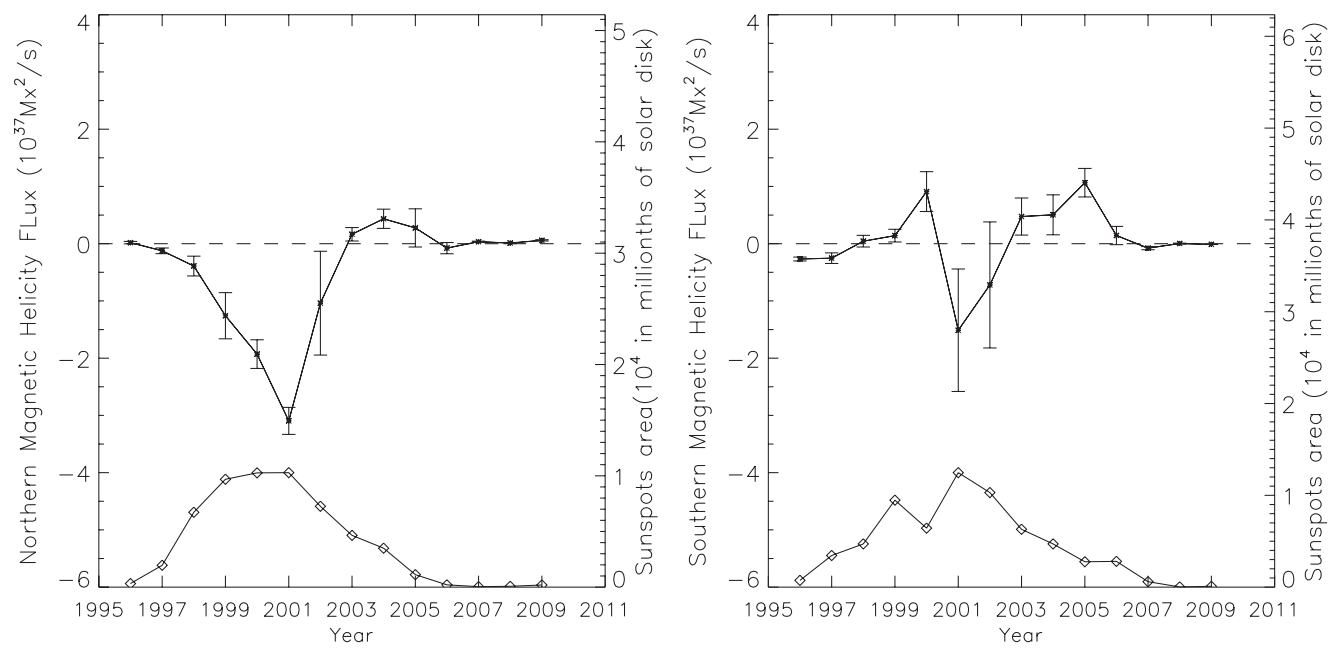

Figure 4. Large scale magnetic helicity fluxes in the northern and southern hemisphere between $-40^{\circ}$ and $40^{\circ}$ latitude over the 23 rd solar cycle. $\sigma$-error bars are shown by vertical lines. The diamond line indicates the total sunspots area of a year in millionths of solar disk over the 23rd solar cycle (readings in the right ordinate). (Yang and Zhang, 2012)

(2010b). By comparing with the reversal models of hemispheric helicity distribution proposed by Choudhuri et al. (2004) and Xu et al. (2009), the new possibilities on the large-scale non-antisymmetric components of magnetic helicity of solar active regions in both hemispheres with solar cycles need to be investigated.

The transfer of magnetic chirality in solar active regions relates to the emerging magnetic flux ropes generated from the subatmosphere. This analysis has been presented based on the calculation on the injection of magnetic helicity in the solar surface. As 
one follows the long-term evolution of accumulated magnetic helicity, it is found that the transfer of reversal magnetic helicity of active regions is a complex process, and it is not monotone with the same sign. It is found that the dominant contribution of helicity mainly occurs in the fast developing stage of active regions.

By comprising with the statistical distribution of the magnetic helicity from the hemispheric trends, it is proposed that the reversal helicity in solar active regions are generated from two possibilities: the local generation in the convection zone and trans-equatorial process in the subatmosphere. Beside the mirror symmetrical reverse of twisted magnetic field in the convection zone, a possibility on the trans-equatorial process with the form of butterfly diagram has been discussed by Zhang (2012) in Figure 3.

To investigate the characteristics of large scale and long term evolution of magnetic helicity with solar cycles, Yang and Zhang (2012) use the method of Local Correlation Tracking (LCT) to estimate the magnetic helicity evolution over the 23rd solar cycle from 1996 to 2009 by using 795 MDI magnetic synoptic charts in Figure 4. The main results are: the hemispheric helicity rule still holds in general, i.e. the large-scale negative (positive) magnetic helicity dominates the northern (southern) hemisphere. However, the large scale magnetic helicity fluxes show the same sign in both hemispheres around 2001 and 2005. The global, large scale magnetic helicity flux over the solar disk changes from negative value at the beginning of the 23rd solar cycle to positive value at the end of the cycle, while the net accumulated magnetic helicity is negative in the period between 1996 and 2009. The consistence between the total injective magnetic helicity and sunspot numbers with the solar cycle has been found also statistically.

Berger and Ruzmaikin (2000) analyzed the helicity injection with solar cycles and found that throughout the 22 year cycle studied(1976-1998) the helicity production in the interior by differential rotation had the correct sign compared to observations of coronal structures - negative in the north and positive in the south. The net helicity flow into each hemisphere over this cycle was approximately $4 \times 10^{46} M x^{2}$. Georgoulis et al. (2009) that accounting for various minor underestimation factors, they estimated a maximum helicity injection of $6.6 \times 10^{45} M x^{2}$ for solar cycle 23. Moreover, Welsch and Longcope (2003) estimated that the hemispheric mutual-helicity flux from the quiet sun is a order of $10^{43} M x^{2}$ for a whole solar cycle.

The diagnosis on the transequatorial connection of magnetic field from active regions in both hemispheric atmospheres is useful for understanding the evolution of large-scale magnetic helicity of the Sun. Pevtsov (2000) found that approximately one-third of all active regions on the Sun exhibit transequatorial loops (TLs), and also found that the reconnected regions have approximately the same rotation rate and tend to appear on certain longitudes, similar to the complexes of activity. In most cases transequatorial interconnected regions have the same handedness of their magnetic field. Chen, Bao and Zhang (2007) pointed out that about $50 \%$ of the active region pairs carry the same current helicity sign and about $50 \%$ of them have the opposite.

Jiang, Choudhuri and Wang (2007) presented a possibility on the origin of TLs linking with the Babcock Leighton dynamo process based on the model of Chatterjee, Nandy, and Choudhuri (2004). They proposed that TLs are visible signatures of poloidal field lines across the equator. Moreover, Yokoyama and Masuda (2009) analyzed TLs observed simultaneously with Yohkoh/SXT and a coronagraph (SOHO/LASCO-C1). SOHO/LASCO-C1 observed loop expansion and eruption at the west solar limb. They proposed a formation mechanism of the TLs that forms between two independent active regions. Yokoyama and Masuda (2010) also found that some TLs were originating with large-scale magnetic fields of the coronal-hole boundary through magnetic reconnection between the active region and a coronal hole. 

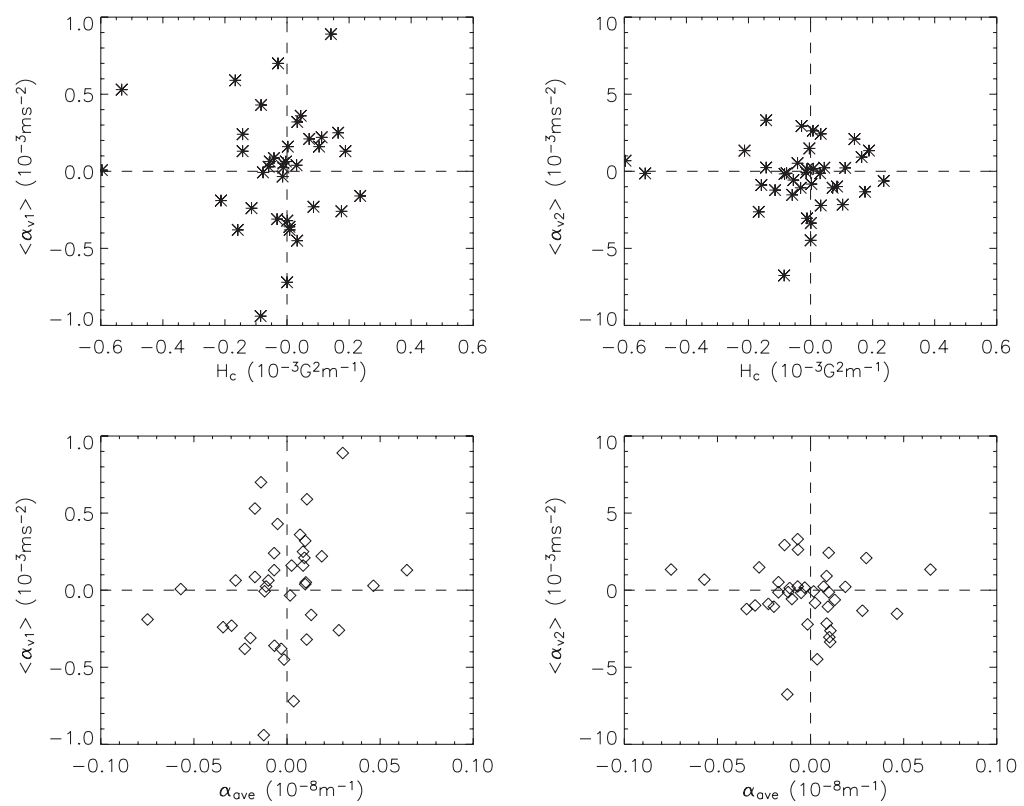

Figure 5. Correlation between photospheric current helicity and subsurface kinetic helicity. Left Top: $\left\langle H_{c}\right\rangle$ and $\left\langle\alpha_{v 1}\right\rangle$; Right Top: $\left\langle H_{c}\right\rangle$ and $\left\langle\alpha_{v 2}\right\rangle$; Left bottom: $\left\langle\alpha_{v 1}\right\rangle$ and $\alpha_{\text {ave }}$; Right bottom: $\left\langle\alpha_{v 2}\right\rangle$ and $\alpha_{\text {ave }}$. (Gao, Zhang, and Zhao, 2009)

\section{Observational kinetic helicity and relationship with magnetic helicity}

An interesting result by Zhao and Kosovichev (2003) and Zhao (2004) showed that the subsurface kinetic helicity inside active regions obtained from Solar Heliospheric Observatory / Michelson Doppler Imager (SOHO/MDI) observations seemed to have a hemispheric preponderance, like what magnetic (or current) helicity observations had shown (Pevtsov et al. 1995; Bao and Zhang 1998). Gao, Zhang and Zhao (2009) presented a comparison between photospheric current helicity and subsurface kinetic helicity in solar active regions in Figure 5. Four parameters are employed: average current helicity $\left\langle B_{z} \cdot(\nabla \times \mathbf{B})_{z}\right\rangle\left(\left\langle H_{c}\right\rangle\right)$, average force-free field factor $\sum(\nabla \times \mathbf{B})_{z} \cdot \operatorname{sign}\left[B_{z}\right] / \sum\left|B_{z}\right|$ $\left(\alpha_{\text {ave }}\right)$ and mean subsurface kinetic helicity $\left\langle\mathbf{v} \cdot(\nabla \times \mathbf{v}) /|\mathbf{v}|^{2}\right\rangle\left(\alpha_{v}\right)$, which is denoted as two different parameters $\left\langle\alpha_{v 1}\right\rangle$ and $\left\langle\alpha_{v 2}\right\rangle$ according to different depths beneath the solar surface. The results show that the signs of $\left\langle H_{c}\right\rangle$ and $\alpha_{\text {ave }}$ have typical hemispheric distribution feature. In contrast, the sign of $\left\langle\alpha_{v 1}\right\rangle$ presents the opposite feature to the above two parameters. For $\left\langle\alpha_{v 2}\right\rangle$, there is not obvious preponderance of the sign in each hemisphere as other three parameters. Although there is opposite hemispheric preponderance between sign of current helicity and that of kinetic helicity at $0-3 \mathrm{Mm}$ beneath the solar surface, the weak correlations between $\left\langle H_{c}\right\rangle$ and $\left\langle\alpha_{v 1}\right\rangle, \alpha_{\text {ave }}$ and $\left\langle\alpha_{v 1}\right\rangle,\left\langle H_{c}\right\rangle$ and $\left\langle\alpha_{v 2}\right\rangle, \alpha_{\text {ave }}$ and $\left\langle\alpha_{v 2}\right\rangle$ do not support that the photospheric current helicity has a cause and effect relation to the kinetic helicity at $0-12 \mathrm{Mm}$ beneath the solar surface.

It is noticed that the high cadence simultaneous observations of vector magnetic field and subsurface flow field makes possible the comparison between two kinds of helicities during the evolution of an active region. Some new achievements in this topic taken by Gao, Zhao and Zhang (2012) has been presented in this proceedings briefly also.

Moreover, Kuzanyan, Pipin and Zhang (2007) and Pipin et al. (2011) showed that the cross-helicity alternates in sign with the solar cycle (so it is zero in the long time average), 
and it changes from negative to positive following the toroidal field. They demonstrated how it is possible to tune such models with respect to account of different effects to reproduce particular features of the observable solar magnetic fields and its helical properties. By means of a quasilinear theory and by numerical simulations, Rüdiger, Kitchatinov and Brandenburg (2011) found the cross helicity and the mean vertical magnetic field to be anti-correlated and predicted that the cross helicity at the solar surface will exceed the value of 1 gauss $\mathrm{km} / \mathrm{s}$. Zhao, Wang and Zhang (2011) used line-of-sight magnetograms and Dopplergrams from SOHO/MDI to determine the distribution of cross helicity in the solar surface and found that the large scale and weak magnetic field (less than 50 $\mathrm{G}$ ) is correlated with the velocity statistically, even if it is a preliminary analysis on the cross helicity in the solar surface.

\section{Discussions}

The study of helicities in the solar active regions is an interesting topic, which relates to the measurements and analysis of solar activities basically.

It should be noticed that the inversion accuracy of Stokes parameters for the measured photospheric vector magnetic field and the resolution of $180^{\circ}$-ambiguity of transverse component of vector magnetic field are still basic questions. From the directorial measurements of magnetic and current helicities taken from the photospheric (vector) magnetograms, one can get the quantities of the transfer rate of magnetic helicity, while one can not get the basic topology of magnetic field in the high solar atmosphere. The measurements of solar vector magnetograms provide a chance to analyze the distribution of partial current helicity density $\left(h_{c z}\right)$ of solar active regions in the solar surface, but it is not the complete helicity density $\left(h_{c}\right)$. The kinetic helicity is an important parameter in the dynamo theory, but it is rarely the observational evidence for a long time. The observational kinetic helicity is normally inferred from the subsurface velocity field based on the the technique of helioseismology. It means that the kinetic helicity depends on the inverting accuracy of helioseismology obviously.

A systematic analysis of magnetic and kinetic helicity in the solar atmosphere is an important chance to know the formation of solar active cycles, and the relationship with possible solar dynamo. Even if amount of samples of photospheric vector magnetograms have been observed at different solar observatories in the last more than 20 years and these data have been used to infer the current helicity of solar active regions, one still finds some slight different helicity results from the different observing sets. Moreover, one also can not get all of vector magnetic fields of solar active regions, due to the absence of the complete observations of vector magnetic fields of the Sun and the evolution with solar cycles. One still does not know more on the kinetic helicity from the observations, due to small amounts of works in this topic only until now. This also means that the relationship between the magnetic and kinetic helicity still needs to be analyzed in more detail.

The solar magnetic and velocity fields are normally measured in the photosphere, while it is far from the formation layers of the solar dynamo and the eruption of flare-CMEs. One still can not know more information on the generation of the magnetic field inside of the Sun, while the twisted magnetic fields in the solar surface have been analyzed in the form of magnetic helicity and the velocity fields in that of kinetic helicity to infer the possible generation of magnetic field and the relationship with solar dynamo. Even if one knows that the formation of flare-CMEs relates to the complex configuration of magnetic fields in the solar surface, while the study on topology of magnetic field in the high solar atmosphere still remains some basic questions. 


\section{Acknowledgements}

This study is supported by the National Natural Science Foundation of China: 10921303, 41174153. The author wold like to thank D. Sokoloff, K. Kuzanyan, T. Sakurai, J. Büchner, Y. Gao, H. Xu, S. Yang, J. Zhao and A. Pevtsov et al. for their kindly cooperations and discussions in the study.

\section{References}

Abramenko, V. I., Wang, T., \& Yurchishin, V. B., 1996, Solar Phys., 168, 75

Bao, S. \& Zhang, H., 1998, Astrophys. J, 496, L43

Bao S., Zhang H., Ai G., \& Zhang M., 1999, Astron. Astrophys., 139, 311

Bao, S. D., Ai, G. X., \& Zhang, H. Q., 2000, JApA, 21, 303

Berger, M. A. \& Field, G. B., 1984, JFM 147, 133

Berger, M. \& Ruzmaikin, A. 2000, JGR, 10510481

Brandenburg, A., Dobler, W., \& Subramanian, K., 2002, AN, 323, 99

Brandenburg, A. \& Subramanian, K., 2005, PhR, 417, 1

Chae, J., 2001, Astrophys. J, 560, L95

Chatterjee, P., Nandy, D., \& Choudhuri, A. R., 2004, A\&A, 427, 1019

Chen J, Bao, S., \& Zhang, H., 2007, Solar Phys., 242, 65C

Choudhuri, A. R., Chatterjee, P., \& Nandy, D., 2004, Astrophys. J, 615, L57

Démoulin, P., \& Berger, M. A., 2003, Solar Phys., 215, 203

Deng, Y., Wang, J., Yan, Y., \& Zhang, J., 2001, Solar Phys., 204, 11

Ding, Y. J., Hong, Q. F., \& Wang, H. Z., 1987, Solar Phys., 107, 221

Gao, Y., Zhang, H., \& Zhao, J., 2009, MNRAS, 394, L79

Gao, Y., Zhao, J., \& ,Zhang, H. 2012, in this proceedings

Georgoulis, M. K., Rust, D. M., Pevtsov, A. A., Bernasconi, P. N., \& Kuzanyan, K. M., 2009, Astrophys. J., 705, L48

Hagino, M. \& Sakurai, T., 2004, PASJ, 56, 831

Hagino, M. \& Sakurai, T., 2005, PASJ, 57, 481

Hale, G. E., Ellerman, F., Nicholsin, S. B., \& Joy, A. H., 1919, Astrophys. J., 49, 153

Jeong, H. \& Chae, J., 2007, Astrophys. J., 671, 1022

Jiang, J., Choudhuri, A. R., \& Wang, J., 2007, Solar Phys., 245, 19

Keinigs, R. K., 1983, PhFl, 26, 2558

Keinigs, R. \& Gerwin, Richard A., 1986, IEEE Transactions, 14, Issue 6, 858

Kleeorin, N. \& Rogachevskii, I., 1999, PhRvE, 59, 6724

Kleeorin, N., Kuzanyan, K., Moss, D., Rogachevskii, I., Sokoloff, D., \& Zhang, H., 2003, A\&A, 409, 1097

Kusano, K., Maeshiro, T., Yokoyama, T., \& Sakurai, T., 2002, Astrophys. J, 577, 501

Kuzanyan, K. M., Lamburt, V. G., Zhang, H., \& Bao, S., 2003, ChJAA, 3, 257

Kuzanyan, K. M., Pipin, V., V., \& Zhang, H., 2007, AdSpR, 39, 1694

LaBonte, B. J., Georgoulis, M. K., \& Rust, D. M., 2007, Astrophys. J, 671, 955

Liu, J. \& Zhang, H., 2006, Solar Phys., 234, 21

Longcope, D. W., Fisher, G. H., \& Pevtsov, A. A., 1998, Astrophys. J, 507, 417

López Fuentes, M. C., Démoulin, P., Mandrini, C. H., \& van Driel-Gesztelyi, L., 2000, Astrophys. $J, 544,540$

Pariat, E., Démoulin, P., \& Berger, M. A., 2005, Astron. Astrophys., 439, 1191

Parker, E., 1955, Astrophys. J, 122, 293

Pevtsov, A. A., 2000, Astrophys. J, 531, 553

Pevtsov, A. A., Canfield, R. C., \& Metcalf, T. R., 1994, Astrophys. J, 425, L117

Pevtsov, A. A., Canfield, R. C., \& Metcalf, T. R., 1995, Astrophys. J, 440, L109

Pevtsov, A., A. \& Latushko, S. M., 2000, Astrophys. J, 528, 999

Pevtsov, A. A., Dun, J. P., \& Zhang, H., 2006, Solar Phys., 234, 203

Pevtsov, A. A., Canfield, R. C., Sakurai, T., \& Hagino, M., 2008, Astrophys. J, 677, 719 
Pipin, V., Kuzanyan, K., Zhang, H., \& Kosovichev, A., 2011, Astrophys. J, 743, 160

Pouquet, A., Frisch, U., \& Leorat, J., 1976, JFM, 77, 321

Rädler, K., \& Rheinhardt, M., 2007, GApFD., 101, 117

Rust, D. M. \& Kumar, A., 1996, Astrophys. J, 464, L199

Rüdiger, G., Pipin, V. V., \& Belvedére, G., 2001, Solar Phys., 198, 241

Rüdiger, G., Kitchatinov, L. L., \& Brandenburg, A., 2011, Solar Phys., 269, 3

Seehafer, N., 1990, Solar Phys., 125, 219

Seehafer, N., 1994, Astron. Astrophys., 284, 593

Sokoloff, D. 2007, Plasma Phys. Control. Fusion, 49, 447

Sokoloff, D., Zhang, H., Kuzanyan, K. M., Obridko, V. N., Tomin, D. N., \& Tutubalin, V. N., 2008, Solar Phys., 248, 17

Su, J. T., Sakurai, T., Suematsu, Y., Hagino, M., \& Liu, Y., 2009, Astrophys. J, 697, L103

Taylor, J. B., 1986, RvMP, 58, 741

Tian, L., Bao, S., Zhang, H., \& Wang, H., 2001, A\&A, 374, 294

Tian, L. \& Alexander, D., 2009, Astrophys. J, 695, 1012

Tiwari, S. K., Venkatakrishnan, P., \& Sankarasubramanian, K., 2009, Astrophys. J, 702, L133

Wang, J., 1996, Solar Phys., 163, 319

Wang, C. \& Zhang, M., 2010, Astrophys. J, 720, 632

Welsch, B. T., \& Longcope, D. W., 2003, Astrophys. J, 588, 620

Woltjer, L., 1958a, Proc. Natl. Acad. Sci. USA, 44, 489

Woltjer, L.: 1958b, Proc. Natl. Acad. Sci. USA, 44, 833

Venkatakrishnan, P., \& Tiwari, S., 2009, Astrophys. J, 706, L114

Xu, H., Gao, Y., Zhang, H., Sakurai, T., Pevtsov, A. A., \& Sokoloff, D., 2007, AdSpR, 39, 1715

Xu, H., Gao, Y., Popova, E. P., Nefedov, S. N., Zhang, H., \& Sokoloff, D. D., 2009, ARep, 53, 160

Xu, H., Gao, Y., Zhang, H., Sakurai, T., Hagino, M., Sokoloff, D., \& Pevtsov, A., 2012, PASJ, 64,54

Yang, S., Zhang, H., \& Büchner, J., 2009, A $\& A$ 502, 333

Yang, S., \& Zhang, H., 20012, Astrophys. J, 758, 61

Yang, X., Zhang, H., Gao, Y., \& Guo, J., Lin, G., 2012, Solar Phys., 280, 165

Yokoyama, M. \& Masuda, S., 2009, Solar Phys., 254, 285

Yokoyama, M. \& Masuda, S., 2010, Solar Phys., 263, 135

Zhang, H., 2001, MNRAS, 326, 57

Zhang, H., 2006a, ChJAA, 6, 96

Zhang, H., 2010, Astrophys. J., 716, 1493

Zhang, H., 2012, MNRAS, 419, 799

Zhang, H. \& Bao, S. 1998, Astron. Astrophys., 339, 880

Zhang, H., Sokoloff, D., Rogachevskii, I., Moss, D., Lamburt, V., Kuzanyan, K., \& Kleeorin, N., 2006, MNRAS, 365, 276

Zhang, H., Sakurai, T., Pevtsov, A., Gao, Y., Xu, H., Sokoloff, D., \& Kuzanyan, K., 2010a, $M N R A S, 402$, L30

Zhang, H., Yang, S., Gao, Y., Su, J., Sokoloff, D. D., \& Kuzanyan, K., 2010b, Astrophys. J., 719,1955

Zhang H., Moss D., Kleeorin, N. Kuzanyan K., Rogachevskii I., Sokoloff D., Gao Y., \& Xu H., 2012, Astron. Astrophys., 751, 47

Zhang, M., 2006b, Astrophys. J, 646, L85

Zhang, Y., Liu, J., \& Zhang, H., 2008, Solar Phys., 247, 39

Zhao, J. 2004, Chap. 5, PhD Thesis, Stanford Univ.

Zhao, J. \& Kosovichev, A. G., 2003, Astrophys. J, 591, 446

Zhao, J., Couvidat, S., Bogart, R. S., Parchevsky K. V., Birch, A. C., Duvall, T. L., Jr., Beck J. G., Kosovichev, A. G., \& Scherrer, P. H. 2012, Solar Phys., 275, 375

Zhao, M. Y., Wang, X. F., \& Zhang, H. Q., 2011, Solar Phys., 270, 23 